\title{
ESTUDIO DE LA DURABILIDAD DE CONCRETOS TERNARIOS CON CENIZA DE BAGAZO DE CAÑA DE AZÚCAR Y HUMO DE SÍLICE
}

\author{
H. A. ARIZA-FIGUEROA ${ }^{1,2}$, R. CROCHE ${ }^{3}$, F. ALMERAYA-CALDERÓN ${ }^{4}$, \\ J. CABRAL ${ }^{4}$, C. GAONA-TIBURCIO ${ }^{4}$, A. C. RAMÍREZ-GARCÍA ${ }^{5}$, \\ R. VILLEGAS-APAEZ ${ }^{6}$, M. A. BALTAZAR-ZAMORA ${ }^{7}$ \\ ${ }^{1}$ Universidad Veracruzana, Doctorado en Ingeniería, FIME, Xalapa, Veracruz, México \\ ${ }^{2}$ GEOTEST S.A. de C.V. - Xalapa, Veracruz, México \\ ${ }^{3}$ Universidad Veracruzana, FIME, Xalapa, Veracruz, México \\ ${ }^{4}$ Universidad Autónoma de Nuevo León. FIME - CIIIA., San Nicolás de los Garza, Nuevo León, México \\ ${ }^{5}$ Universidad Veracruzana, Facultad de Ingeniería, Cd. Mendoza, Veracruz, México \\ ${ }^{6}$ Ingenio Mahuixtlán S.A de C.V, Loc., Mahuixtlán, Coatepec, Veracruz, México \\ ${ }^{7}$ Universidad Veracruzana, Facultad de Ingeniería Civil - Xalapa, Circ. G. Aguirre Beltrán S/N, Lomas del Estadio, CP \\ 91000, Xalapa, Veracruz, México.
}

\begin{abstract}
RESUMEN
La fabricación del Cemento Portland (CP), representa una de las mayores fuentes de emisiones de $\mathrm{CO} 2$. Hay diversas alternativas para evitar el deterioro del medio ambiente, entre ellas el uso de materiales alternativos al CP, que pueden ser de origen industrial y agroindustrial. Esta investigación evalúa las propiedades físicas, mecánicas y de durabilidad de concretos ternarios elaborados con sustitución parcial del Cemento Portland (CP) por combinaciones de Ceniza de Bagazo de Caña de Azúcar (CBCA) y Humo de Sílice (HS), en un 10, 20 y 30\%. El diseño de las mezclas de concretos convencional y ternarios fue acorde al ACI 211.1, realizando el curado en medios sulfatados, soluciones al 3.5\% de Na2SO4 y MgSO 4 , simulando cuando el concreto de Obras Civiles nuevas son colocados en medios de contacto con la presencia de estos agentes agresivos. Los ensayos para determinar las propiedades mecánicas y de durabilidad se realizaron en base a las normas ONNCCE y ASTM. Los resultados muestran un buen desempeño en los concretos ternarios con sustitución parcial de $10 \%, 20 \%$ y $30 \%$ de combinaciones de CBCA-HS por CP.
\end{abstract}

Palabras clave: Concretos Ternarios, CBCA, HS, Durabilidad, Propiedades Físicas y Mecánicas.

\begin{abstract}
The manufacture of Portland Cement (PC) represents one of the largest sources of $\mathrm{CO} 2$ emissions. There are several alternatives to avoid environmental deterioration, including the use of alternative materials to the PC, which can be of industrial and agroindustrial origin. This research evaluates the physical, mechanical and durability properties of ternary concretes made with partial replacement of Portland Cement (PC) by combinations of Sugar Cane Bagasse Ash (SCBA) and Silica Fume (SF), in a 10, 20 and $30 \%$. The design of conventional and ternary concrete mixtures was in accordance with ACI 211.1, curing in sulfated media, 3.5\% solutions of $\mathrm{Na} 2 \mathrm{SO} 4$ and $\mathrm{MgSO} 4$, simulating when new Civil Works concrete is placed in contact media with the presence of these aggressive agents. The tests to determine the mechanical and durability properties were performed based on the ONNCCE and ASTM standards. The results show a good performance in ternary concrete with partial replacement of $10 \%, 20 \%$ and $30 \%$ of combinations of SCBA-SF by CP.
\end{abstract}

Keywords: Ternary Concrete, CBCA, HS, Durability, Physical and Mechanical Properties. 


\section{RESUMO}

A fabricação de Cimento Portland $(\mathrm{CP})$ representa uma das maiores fontes de emissões de $\mathrm{CO}_{2}$. Existem várias alternativas para evitar a deterioração ambiental, incluindo o uso de materiais alternativos à $\mathrm{CP}$, que podem ser de origem industrial e agroindustrial. Esta pesquisa avalia as propriedades físicas, mecânicas e de durabilidade de concretos ternários confeccionados com a substituição parcial do Cimento Portland (CP) por combinações de Cinza de Bagaço de Cana de açucar (CBCA) e Fumaça de Sílica (FS), em 10, 20 e 30\%. O projeto de misturas convencionais e ternárias de concreto foi de acordo com ACI 211.1, cura em meios sulfatados, soluções a 3,5\% de $\mathrm{Na}_{2} \mathrm{SO}_{4} \mathrm{e}$ $\mathrm{MgSO}_{4}$, simulando quando novos concretos da Civil Works são colocados em meios de contato com a presença de esses agentes agressivos. Os testes para determinar as propriedades mecânicas e de durabilidade foram realizados com base nos padrões ONNCCE e ASTM. Os resultados mostram um bom desempenho em concreto ternário com substituição parcial de 10\%, $20 \%$ e $30 \%$ das combinações de CBCA-FS por CP.

Palavras-chave: Concreto Ternário, CBCA, HS, Durabilidade, Propriedades Físicas e Mecânicas

\section{INTRODUCCIÓN}

Por muchos años se ha considerado al cemento como la parte fundamental de la construcción. En conjunto con el cemento y el acero forman el sistema conocido como concreto reforzado. El uso del concreto como elemento constructivo ha estado presente en multitud de estructuras y edificaciones desde los albores del Imperio Romano hasta nuestros días. Paralelamente han ido evolucionando con él, tanto los elementos básicos de las obras en las que se ha utilizado este material como la propia puesta en obra del mismo (Cordero, at.al., 2012; Baltazar, 2000; Baltazar, 2016; A.E. Landa et.al 2018; G. Santiago et.al 2016). El concreto es la segunda materia prima más consumida en la Tierra después del agua, y es, con mucho, el material de construcción más utilizado en todo el mundo (Y.L. Yaphary, at.al., 2017; A. Landa et.al, 2019; D. Garcia et.al, 2019, M. Ruiz, 2019). La gran popularidad del concreto se debe a sus excelentes características, como gran durabilidad, resistencia, etc. No obstante, su uso también acarrea unos grandes costes medioambientales, destacando particularmente, la enorme cantidad de energía consumida y $\mathrm{CO}_{2}$ liberado durante su fabricación. Además, la obtención de áridos y materias primas necesarias para la obtención del cemento, puede implicar la destrucción de ciertos hábitats, así como causar problemas de contaminación en el aire y agua de la zona (Cagiao, J, at.al., 2012).

Para mitigar los efectos en el medio ambiente, se ha empezado a hacer uso de materiales alternativos al cemento portland, los cuales han logrado un posicionamiento al dar magníficos resultados en sus propiedades primarias, tales materiales como ceniza volante, ceniza de bagazo de caña de azúcar (A.E. Landa, 2018; A. Landa et.al, 2019; A.E. Landa, 2018; H. Ariza, 2019; Ojeda, 2018, A.E. Landa, 2018; D. Garcia et.al, 2019).

\section{PROCEDIMIENTO}

En la presente investigación se realizaron cilindros de concretos ecológicos ternarios, a base de cemento portland y sustituciones parciales de ceniza de bagazo de caña de azúcar (CBCA) y humo de sílice (HS), se elaboró el espécimen con concreto convencional como testigo, con la intención de utilizarlo para comparación con los concretos ecológicos ternarios. Durante un periodo de 28 días, se determinaron sus propiedades en estado fresco, la resistencia a la compresión, módulo de elasticidad estático y resistividad eléctrica como parámetro de durabilidad, el curado de las 4 mezclas de concreto fue en dos medios sulfatados, soluciones al 3.5\% de $\mathrm{Na}_{2} \mathrm{SO}_{4}$ y $\mathrm{MgSO}_{4}$. El objetivo de este proyecto de investigación es comprobar que es factible la fabricación de concretos reduciendo en forma 
considerable el uso del cemento Portland, al reutilizar desechos como lo es la CBCA y el HS, buscando generar beneficios económicos y medioambientales, y demostrando, además, que estas sustituciones en altos volúmenes aportan grandes mejoras a las propiedades del concreto.

\subsection{Diseño, dosificación y nomenclatura de las mezclas para los concretos ecológicos ternarios}

La dosificación de las mezclas se utilizó el método del Instituto Americano del Concreto ACI 211.1 [ACI, 2004], metodología que considera el revenimiento, la resistencia a la compresión de diseño, y las características físicas de los agregados, las cuales se realizaron de acuerdo a las normas ASTM [ASTM, 2007; ASTM, 2014; ASTM, 2015; ASTM, 2015; ASTM, 2016]. La Tabla 1, resume las características físicas de los agregados gruesos y finos que se utilizaron en la presente investigación.

Tabla 1. Resumen de la caracterización física de los agregados.

\begin{tabular}{|c|c|c|}
\hline Propiedades físicas de los materiales & $\begin{array}{c}\text { Agregado } \\
\text { grueso }\end{array}$ & Agregado fino \\
\hline $\begin{array}{c}\text { Densidad relativa aparente saturada y } \\
\text { superficialmente seca (Dr SSS) gr/cm }\end{array}$ & 2.60 & 2.20 \\
\hline Masa Volumétrica Suelta (MVS) Kg/m ${ }^{3}$ & 1332.00 & 1442.00 \\
\hline Absorción \% (A) & 1.70 & 1.80 \\
\hline Módulo de finura & $19 \mathrm{~mm}$ & 2.94 \\
\hline Tamaño nominal & & \\
\hline
\end{tabular}

Se utilizó la relación agua/cemento $=0.65$. La mezcla de referencia $(\mathrm{REF})$ es a base de $100 \%$ de cemento portland compuesto (CPC). En las mezclas continuas se realizaron en sustituciones parciales de cemento portland de 10\%, 20\% y 30\%, de combinaciones de CBCA-HS. Para el análisis de resultados se planteó la siguiente nomenclatura para las mezclas de estudio. La tabla 2 presenta el proporcionamiento de las cuatro mezclas de estudio.

Donde:

REF.- Mezcla 100\% CPC.

05,10,15 SCBA y/o FS.- porcentaje de sustitución de Ceniza de Bagazo de Caña de Azúcar y Humo de Sílice.

Tabla 2. Dosificación de los especímenes de estudio para $1 \mathrm{~m}^{3}$.

\begin{tabular}{|c|c|c|c|c|c|c|}
\hline Mezcla & $\begin{array}{c}\text { Cemento } \\
(\mathbf{k g})\end{array}$ & $\begin{array}{c}\text { Ceniza de } \\
\text { Bagazo de } \\
\text { Caña }(\mathbf{K g})\end{array}$ & $\begin{array}{c}\text { Humo } \\
\text { de Sílice } \\
(\mathbf{k g})\end{array}$ & $\begin{array}{c}\text { Agua } \\
\mathbf{( K g )}\end{array}$ & $\begin{array}{c}\text { Agregad } \\
\mathbf{o f i n o} \\
(\mathbf{K g})\end{array}$ & $\begin{array}{c}\text { Agregado } \\
\text { grueso } \\
(\mathbf{k g})\end{array}$ \\
\hline REF & 314.61 & - & - & 197.79 & 770.11 & 886.00 \\
\hline 10(05SCBA05FS) & 284.06 & 15.77 & 15.77 & 200.60 & 770.76 & 888.64 \\
\hline 20(10SCBA10FS) & 252.51 & 31.55 & 31.55 & 302.16 & 772.64 & 884.22 \\
\hline 30(15SCBA15FS) & 220.96 & 43.35 & 43.35 & 206.71 & 772.55 & 880.73 \\
\hline
\end{tabular}

\subsection{Características de los especímenes de ensayo}

Se diseñaron especímenes cilíndricos de las siguientes dimensiones:

Tabla 3. Dimensiones de los especímenes.

\begin{tabular}{|l|l|}
\hline Diámetro & $10 \mathrm{~cm}$ \\
\hline Altura & $20 \mathrm{~cm}$ \\
\hline Sección transversal & $78.54 \mathrm{~cm}$ \\
\hline
\end{tabular}



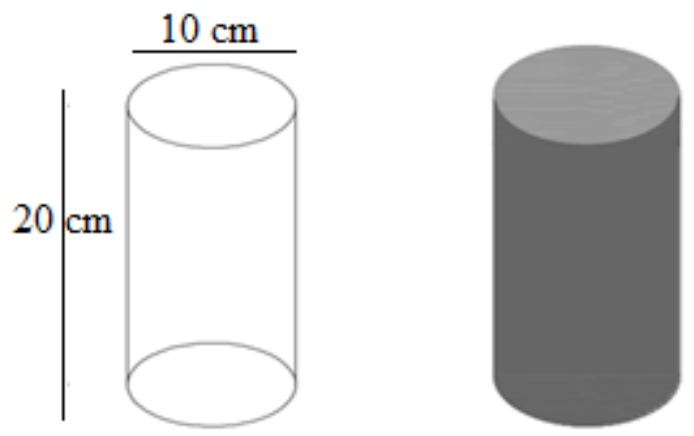

Figura 1. Especificaciones de los especímenes de ensayo.

\subsection{Nomenclatura de especímenes para los ensayes}

Para la facilitar la identificación de los especímenes se realizaron nomenclaturas, para los ensayes de resistencia a la compresión, resistividad y módulo de elasticidad expuestos a los medios agresivos, sulfato de sodio y sulfato de magnesio.

Tabla 4. Nomenclatura de especímenes de ensaye a compresión y Resistividad.

\begin{tabular}{|c|c|}
\hline Nomenclatura & Ensayo \\
\hline MC-3-C/R y MC-4-C/R & F'c y Resistividad a 28 días \\
\hline M1-3-C/R y M1-4-C/R & F'c y Resistividad a 28 días \\
\hline M2-3-C/R y M2-4-C/R & F'c y Resistividad a 28 días \\
\hline M3-3-C/R y M3-4-C/R & F'c y Resistividad a 28 días \\
\hline
\end{tabular}

Donde:

REF.- Mezcla 100\% CPC.

05,10,15 SCBA y/o FS.- porcentaje de sustitución de Ceniza de Bagazo de Caña de Azúcar y Humo de Sílice.

3 y 4.- Medio Agresivo $\left(\mathrm{Na}_{2} \mathrm{SO}_{4} \mathrm{Y} \mathrm{MgSO}_{4}\right)$.

CR.- Ensayos Compresión y Resistividad.

Tabla 5. Nomenclatura de especímenes del ensaye de Módulo de elasticidad.

\begin{tabular}{|c|c|}
\hline Nomenclatura & \multicolumn{1}{|c|}{ Ensayo } \\
\hline MC-3-M y MC-4-M & Módulo de E. a 28 días \\
\hline M1-3-M y M1-4-M & Módulo de E. a 28 días \\
\hline M2-3-M y M2-4-M & Módulo de E. a 28 días \\
\hline M3-3-M y M3-4-M & Módulo de E. a 28 días \\
\hline
\end{tabular}

Donde:

REF.- Mezcla 100\% CPC.

05,10,15 SCBA y/o FS.- porcentaje de sustitución de Ceniza de Bagazo de Caña de Azúcar y Humo de Sílice.

3 y 4.- Medio Agresivo $\left(\mathrm{Na}_{2} \mathrm{SO}_{4} \mathrm{Y} \mathrm{MgSO}_{4}\right)$.

M.- Módulo de elasticidad. 


\section{RESULTADOS}

Los resultados de los ensayes de cilindros a base de concretos ternarios se muestran a continuación.

\subsection{Propiedades físicas del concreto en estado fresco}

De acuerdo a los ensayos de la normativa ONNCCE y ASTM, se determinaron las características del concreto en estado fresco Revenimiento [ONNCCE, 2010], Temperatura [ASTM, 2008], Masa volumétrica [ONNCCE, 2010]. Se muestran temperaturas de las diferentes mezclas, estas oscilan entre los $22.5^{\circ} \mathrm{C}$ y $24{ }^{\circ} \mathrm{C}$, encontrándose dentro de las especificaciones de temperatura de $13^{\circ} \mathrm{C}$ a $38^{\circ}$. Se presentó una absorción mayor de agua que la mezcla control, esto se refleja en la disminución del revenimiento, entre $1,1.5$ y $2 \mathrm{~cm}$, para las mezclas con sustitución de 10, 20 y $30 \%$ de combinación de Ceniza de Bagazo de Caña de Azúcar y Humo de Sílice, respectivamente, por lo que la trabajabilidad fue relativamente menor. Por su parte, la masa unitaria de los concretos sustentables ternarios se mantuvo por arriba de los $1,900 \mathrm{~kg} / \mathrm{m} 3$ que representa de un concreto para uso estructural. Lo anterior se resume en la siguiente tabla.

Tabla 6. Propiedades físicas de las mezclas de concreto ecológicos en estado fresco.

\begin{tabular}{|c|c|c|c|}
\hline Mezcla & $\begin{array}{c}\text { Temperatura } \\
\left({ }^{\circ} \mathbf{C}\right)\end{array}$ & $\begin{array}{c}\text { Revenimiento } \\
(\mathbf{c m})\end{array}$ & $\begin{array}{c}\text { Masa Unitaria } \\
\left(\mathbf{k g} / \mathbf{m}^{\mathbf{3}}\right)\end{array}$ \\
\hline REF & 24.00 & 7.00 & 2345.83 \\
\hline 10(05SCBA05FS $)$ & 23.50 & 6.00 & 2307.29 \\
\hline 20(10SCBA10FS $)$ & 23.50 & 5.50 & 2301.04 \\
\hline 30(15SCBA15FS $)$ & 22.50 & 5.00 & 2276.04 \\
\hline
\end{tabular}

\subsection{Resistencia a la compresión}

El ensayo de Resistencia a compresión se realizó acorde a la normativa de la ONNCCE [ONNCCE, 2014], los resultados se presentan en la figura 2 , se puede observar una mayor resistencia a la compresión los concretos con sustitución de 10\% de la combinación Ceniza de Bagazo de Caña de Azúcar y Humo de Sílice con resistencias de 335.39 y $316.92 \mathrm{~kg} / \mathrm{cm}^{2}$ y porcentajes de 123 y $115 \%$ respecto a la mezcla de diseño. Se aprecia también considerablemente que los concretos con sustitución del $30 \%$ fueron los más desfavorables en su comportamiento mecánico con 232.73 y $217.07 \mathrm{~kg} / \mathrm{cm}^{2}$, y porcentajes de 84 y $78 \%$, curados en sulfato de sodio y sulfato de magnesio respectivamente, quedando por debajo de la resistencia para la cual fueron diseñadas.

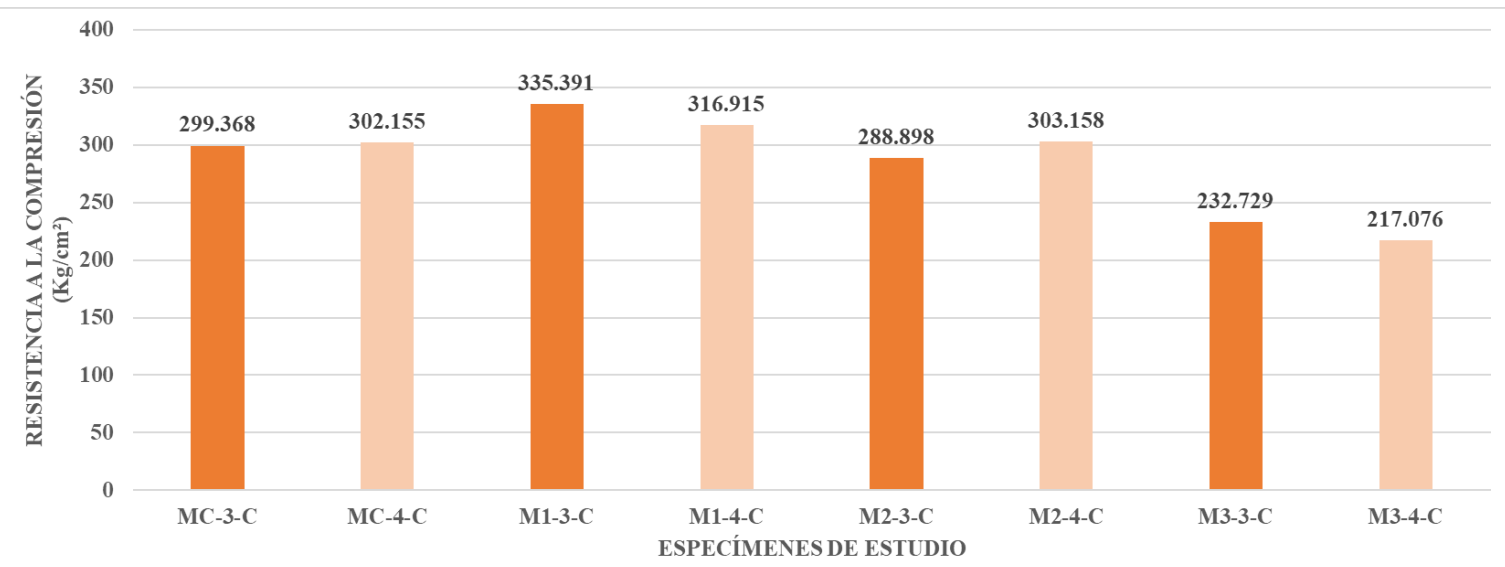

Figura 2. Resistencia a la compresión de Concretos Ecológicos a 28 días 


\subsection{Módulo de elasticidad}

Los resultados muestran que el concreto que mejor se comportó fue el de sustitución de $10 \%$ curado en sulfato de magnesio. Y el más desfavorable fue el de sustitución de 30\% curado en sulfato de sodio.

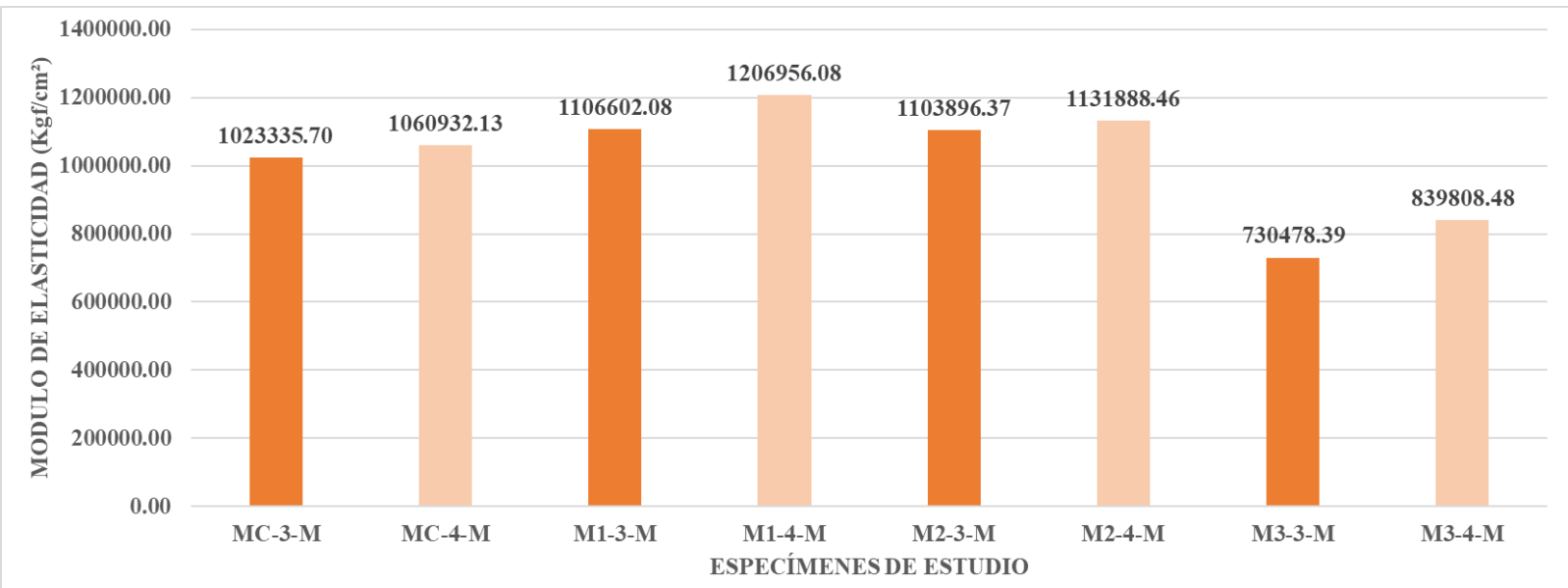

Figura 3. Módulo de elasticidad de concretos ecológicos ternarios.

\subsection{Resistividad eléctrica}

En el día 28 se observa una gran diferencia de resistividad eléctrica entre los especímenes ensayados, el concreto ternario con $30 \%$ de sustitución de ceniza de bagazo de caña y humo de sílice presenta el mejor comportamiento al ensaye de resistividad eléctrica con valores de 35.03 y $34.83 \mathrm{~K} \Omega-\mathrm{cm}$, curadas en sulfato de sodio y sulfato de magnesio respectivamente, así mismo se encuentra en la zona de riesgo moderado. Es importante mencionar que todos los concretos ternarios a la edad de 28 días ya se encuentran en la zona de riesgo moderado.

Revisando investigaciones, las mezclas de concretos ternarios de ceniza de bagazo de caña de azúcar y humo de sílice con sustituciones de 20 y $30 \%$ a una edad de 28 días, alcanzan una resistividad arriba de $10 \mathrm{~K} \Omega-\mathrm{cm}$ por lo que están dentro de la zona de riesgo moderado (Landa Gómez, at.al., 2018).

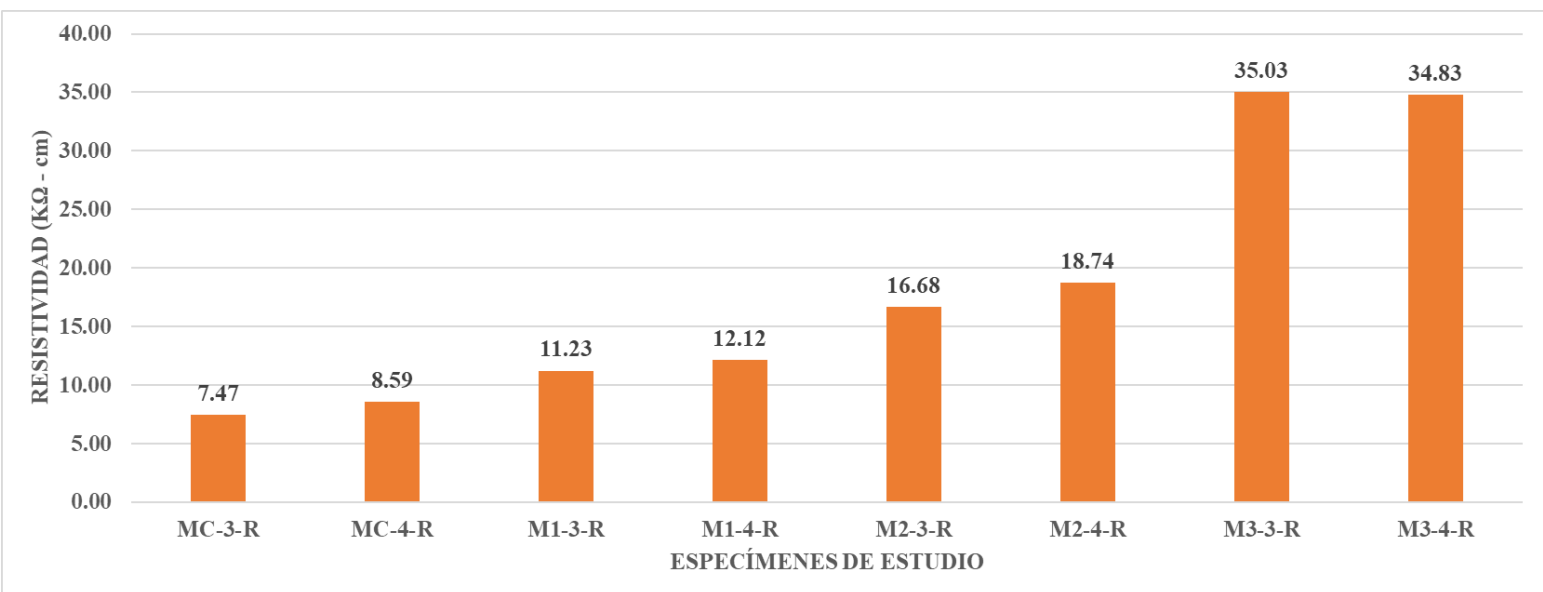

Figura 3. Resistividad de los especímenes a 28 días

\section{CONCLUSIONES}

Los Concretos Ecológicos base sustitución parcial del Cemento Portland por Ceniza de Bagazo de Caña de Azúcar y Humo de Sílice presentan un mejor desempeño en su Resistencias a Compresión y en el Módulo de Elasticidad en combinaciones de 10 y 20\%, El Concreto Ecológico con una sustitución de 
30\% de la combinación Ceniza de Bagazo de Caña de Azúcar y Humo de Sílice presento menores valores de Resistencia a Compresión, pero mayores a $200 \mathrm{~kg} / \mathrm{cm}^{2}$, que es una resistencia a la compresión que puede ser utilizada para construcción de Obras menores. Sin embargo, al someterlo al ensaye de resistividad presentó los mejores valores. Los resultados demuestran la potencialidad del uso de Concretos Ecológicos que presentan buen desempeño al ser expuestos a Sulfatos lo que contribuye en un desarrollo sustentable en el área de la construcción, al disminuir el uso del Cemento Portland hasta en un 20\% para Obras Civiles mayores como Puentes, Edificios, Carreteras, Plantas de Agua de Tratamiento, etc., y de hasta un $30 \%$ en Obras menores, lo que impactaría directamente en la disminución de las emisiones de $\mathrm{CO}_{2}$ debido a la producción del Cemento Portland.

\section{AGRADECIMIENTOS}

M.A. Baltazar-Zamora, et. al., agradecen a PRODEP por el Apoyo otorgado por la SEP, al Cuerpo Académico UV-CA-458 "Sustentabilidad y Durabilidad de Materiales para la Infraestructura Civil", en el marco de la Convocatoria 2018 para Fortalecimiento de Cuerpos Académicos con IDCA 28593.

\section{REFERENCIAS}

ACI. Proporcionamiento de Mezclas, Concreto normal, pesado y masivo ACI 211.1, p. 29. Ed. IMCYC, México (2004).

ASTM International. (2007), ASTM C29 / C29M-07-Standard Test Method for Bulk Density ("Unit Weight”) and Voids in Aggregate.

ASTM International. (2014), ASTM C136 / C136M -14-Standard Test Method for Sieve Analysis of Fine and Coarse Aggregates.

ASTM International. (2015), ASTM C127-15-Standard Test Method for Relative Density (Specific Gravity) and Absorption of Coarse Aggregate.

ASTM International. (2015), ASTM C128-15-Standard Test Method for Relative Density (Specific Gravity) and Absorption of Fine Aggregate.

ASTM International. (2016), ASTM C33/C33M-16e1-Standard Specification for Concrete Aggregates.

ASTM International. (2008) ASTM C1064/C1064M - 08 Standard test method for temperature of freshly Mixed Hydraulic-Cement Concrete.

Baltazar-Zamora M.A. (2000), Corrosión por Sulfatos en Concreto Reforzado, Tesis de Maestría, Facultad de Ingeniería, U.A.CH., Chihuahua, Chih. México.

Baltazar-Zamora M.A., Santiago, G., Moreno V., Croche R., De la Garza M., Estupiñan, F., Zambrano, P., Gaona, C. (2016). Electrochemical Behaviour of Galvanized Steel Embedded in Concrete Exposed to Sand Contaminated with NaCl. Int. J. Electrochem. Sci. 11, 10306-10319, doi: 10.20964/2016.12.28. Cagiao, J., Gómez, B., Doménech, J. L., Gutiérrez, S., Gutiérrez, H., Martínez, F., \& González, M. B. (2010). Huella ecológica del cemento: cálculo de la huella ecológica de una industria cementera y propuesta de medidas de ingeniería sostenible destinadas a su reducción. Universidad da Coruña, ETSICCP da Coruña, Fundación de la Ingeniería Civil de la Coruña, Laboratorio de Ingeniería Sostenible. Coruña.

Cordero, Á.F.N., Abrio, M.T.R., \& Maqueda, M. J. R. (2012). El hormigón: Historia, antecedentes en obras y factores identificativos de su resistencia. Tecnología y desarrollo, 10, 13.

García D.L, Cabral J.A, Gaona C., Almeraya F., Ramírez A.C., Croche R., Baltazar-Zamora M.A. (2019) "Influencia del medio ambiente de exposición y tipo de curado en la cinética de corrosión de concreto reforzado" Memorias del XXXIV Congreso Nacional de la Sociedad Mexicana de Electroquímica, 12th Meeting of the Mexican Section of the Electrochemical Society, SMEQ, Querétaro (México). 
García D.L, Cabral J.A, Gaona C., Almeraya F., Ramírez A.C., Villegas R., Marquez S., BaltazarZamora M.A. (2019). "Eficiencia anticorrosiva del acero AISI 304 como refuerzo en concretos ecológicos ternarios base CBCA-HS expuestos a sulfato de magnesio". Memorias del XXXIV Congreso Nacional de la Sociedad Mexicana de Electroquímica, 12th Meeting of the Mexican Section of the Electrochemical Society, SMEQ, Querétaro (México).

Hilda Amalia Ariza Figueroa, R. Croche B, C. Gaona-Tiburcio, F. Almeraya-Calderón, A.C. RamírezGarcía, R. Villegas-Apaez, S. Marquez-Montero, M.A. Baltazar-Zamora. Evaluación de la corrosión del acero galvanizado y acero AISI 304 embebidos en concretos ecológicos con sustitución de CPC por 10, 20 y 30\% de CBCA-HS expuestos a Na2SO4 al 3.5\%. SMEQ 2019.

Landa A.E., Croche R., Márquez S., Villegas R., Ariza H.A., Estupiñan F., Gaona C., Almeraya F., Baltazar-Zamora M.A. (2018). Corrosion Behavior 304 and 316 Stainless Steel as Reinforcement in Sustainable Concrete Based on Sugar Cane Bagasse Ash Exposed to $\mathrm{Na}_{2} \mathrm{SO}_{4}$, ECS transaction, 84, 179188

Landa A.E., Croche R., Márquez S., Galván R., Gaona C., Almeraya F., Baltazar-Zamora M.A. (2018). Correlation of Compression Resistance and Rupture Module of a Concrete of Ratio $\mathrm{w} / \mathrm{c}=0.50$ with the Corrosion Potential, Electrical Resistivity and Ultrasonic Pulse Speed, ECS transactions, 84, 217-227 Landa A.E., Croche R., López O.M., Galván R., Cabral J.A., Gaona C., Almeraya F., Baltazar-Zamora M.A. (2018). Corrosion Behavior of AISI 316 Stainless Steel As Reinforcement in Ternary Sustainable Concrete Based on SCBA-SF Exposed in Seawater, ECS Meeting Abstracts, MA2018-02 584-584

Landa A.E., Castillo S., Reyes J.A., Villegas R., Galván R., Cabral J.A., Gaona C., Almeraya F., Baltazar-Zamora M.A. (2018). Evaluation of the Corrosion of AISI 304 Stainless Steel Embedded in Sustainable Concrete with High Volumes of SCBA-SF Exposed in Marine Environment, ECS Meeting abstracts, MA2018-02 587

Landa A., Cabral J., Gaona C., Almeraya F., Ramírez A.C., Ruiz M., Malpica R., Márquez S., BaltazarZamora M.A. (2019) "Evaluación electroquímica del acero inoxidable AISI 316 embebido en concretos sustentables base ceniza de bagazo de caña de azúcar expuestos a un medio marino" Memorias del XXXIV Congreso Nacional de la Sociedad Mexicana de Electroquímica, 12th Meeting of the Mexican Section of the Electrochemical Society, SMEQ, Querétaro (México).

Landa A., Landa A., Rangel J.M., Almeraya F., Croche B., Baltazar-Zamora M.A., (2019). "Comportamiento de la corrosión del acero AISI 316 como refuerzo en concretos ternarios sustentables con sustitución parcial de CPC en 10\%, 20\% y 30\% de CBCA-HS inmersos en agua de mar". Memorias del XXXIV Congreso Nacional de la Sociedad Mexicana de Electroquímica, 12th Meeting of the Mexican Section of the Electrochemical Society, SMEQ, Querétaro (México).

Norma Mexicana, N.M.X.C-083-ONNCCE-2002. Determinación de la Resistencia a la Compresión de Especímenes - Método de Ensayo. Organismo Nacional de Normalización y Certificación de la Construcción y Edificación.

Norma Mexicana, N.M.X.C-105-ONNCCE-2010. Concreto Hidráulico Ligero Para uso EstructuralDeterminación de la Masa Volumétrica. Organismo Nacional de Normalización y Certificación de la Construcción y Edificación.

Norma Mexicana, N.M.X.C-156-ONNCCE-2010. Concreto Hidráulico - Determinación Del Revenimiento En El Concreto Fresco. Organismo Nacional de Normalización y Certificación de la Construcción y Edificación.

Ojeda Farías, O., Baltazar Zamora, M., \& Mendoza Rangel, J. (2018). Influencia de la inclusión de ceniza de bagazo de caña de azúcar sobre la compactación, CBR y resistencia a la compresión simple de un material granular tipo subrasante. Revista ALCONPAT, 8(2), 194-208. doi: http://dx.doi.org/10.21041/ra.v8i2.282

Ruíz M., Landa A., Cabral J.A., Gaona C., Almeraya F., Malpica R., Márquez S., Baltazar M.A., (2019) "Influencia del tipo de acero en el comportamiento de la corrosión de concretos sustentables ternarios 
elaborados con ceniza de bagazo de caña de azúcar y humo de sílice expuestos a sulfato de sodio" Memorias del XXXIV Congreso Nacional de la Sociedad Mexicana de Electroquímica, 12th Meeting of the Mexican Section of the Electrochemical Society, SMEQ, Querétaro (México).

Santiago, G., Baltazar-Zamora M.A., Galván, R., López, L.D., Zapata, F., Zambrano, P., A. Gaona, C. and Almeraya, F. (2016), Electrochemical evaluation of reinforcement concrete exposed to soil type sp contaminated with sulphates. Int. J. Electrochem. Sci. 11,4850-4864, doi: 10.20964/2016.06.31

Y.L. Yaphary, R.H.W. Lam, D. Lau. (2017), Chemical technologies for modern concrete production Procedia Eng., 172, pp. 1270 